\title{
Erratum
}

\section{Selective Fries Rearrangement Catalyzed by Zinc Powder}

Satya Paul,* Monika Gupta Synthesis 2004, 1789.

Some incorrect values were given for reaction times and temperatures. The authors apologize for the errors in Table 1, entry 1 and in the text referring to that entry. The correct reaction time for the conversion of phenyl acetate to 2-hydroxyacetophenone under microwave conditions was 2 minutes rather than 3 minutes, and under oil bath heating at $65^{\circ} \mathrm{C}$, the time required was only 1.5 hours instead of 6 hours.

In addition, the column header indicating the units of time for the microwave reactions was inadvertently changed to hours; the correct unit is minutes. The editorial office apologizes for this mistake. 